Original

\title{
Odontogenic cysts: a retrospective study of 1227 cases in an Iranian population from 1987 to 2007
}

\author{
Mohammad J. Sharifian') and Maryam Khalili2) \\ 1)Students' Scientific Research Center, Tehran University of Medical Sciences, Tehran, Iran \\ 2)Department of Oral and Maxillofacial Pathology, Faculty of Dentistry, \\ Tehran University of Medical Sciences, Tehran, Iran
}

(Received 8 January and accepted 19 July 2011)

\begin{abstract}
Odontogenic cysts constitute a major part of oral pathological lesions, but little is known about their distribution patterns in various populations. In this retrospective study, patient records from the archive of the Department of Oral and Maxillofacial Pathology at the Faculty of Dentistry affiliated to the Tehran University of Medical Sciences were reviewed. Cases diagnosed as odontogenic cyst from 1987 to 2007 were selected and histopathologically re-evaluated to confirm the diagnosis. Patients' demographic information (age and sex) and location of the lesion were recorded and analyzed using descriptive statistics. From a total of 8529 files reviewed, the diagnosis of odontogenic cyst was confirmed in 1227 cases (14.4\%). The overall male to female ratio was 1.33:1. The patients' age ranged from 3 to 84 years with a mean of 28 years. $49 \%$ of cysts were found in maxilla and $50.4 \%$ in mandible. Radicular cyst was the most common type followed by dentigerous cyst, odontogenic keratocyst, residual cyst and paradental cyst. Lateral periodontal cyst, eruption cyst, glandular odontogenic cyst and gingival cyst of adults were also reported. The distribution pattern of odontogenic cysts in Iran is relatively similar to that in other parts of the world. The importance of complete clinical reports for final diagnosis of these lesions and routine follow-up examinations is emphasized. ( $\mathrm{J}$ Oral Sci 53, 361-367, 2011)
\end{abstract}

Correspondence to Dr. Maryam Khalili, Department of Oral and Maxillofacial Pathology, Faculty of Dentistry, Tehran University of Medical Sciences, 1417614411, Enghelab St., Ghods Ave., Tehran, Iran

Tel: +98-2188497406

E-mail: mkhalili@tums.ac.ir
Keywords: odontogenic cysts; jaw lesions; epidemiology; demographic study.

\section{Introduction}

Several classifications have been published in order to better define and understand odontogenic cysts (OCs) (1). Based on their origin and pathogenesis, OCs are best classified as inflammatory and developmental. Inflammatory cysts develop as a result of inflammation, but the inciting factors that initiate the formation of developmental cysts remain unknown (2).

The clinical signs and symptoms as well as biologic behavior of these lesions related to recurrence, aggressive or expansive growth, and malignant potential, differs by type. Some entities like odontogenic keratocyst $(\mathrm{OKC})$, recently renamed as keratocystic odontogenic tumor by the World Health Organization (WHO), have a high recurrence rate and show features of neoplastic and aggressive growth, while others are considered as completely benign lesions with no obvious clinical signs and excellent outcomes $(2,3)$.

While clinical and radiographic assessments are necessary, the diagnosis is ultimately dependent on histopathologic findings (4). In addition, reports of carcinomas arising from the cystic wall (5) highlight the need for biopsies of these lesions. However, there is no definite certainty that all these lesions can be biopsied or that all patients seek routine dental and oral examinations.

One of the main aspects of diseases is their demographic and distribution pattern, which leads to better understanding and further clinical implications. Such information regarding odontogenic cysts could be obtained from several sources such as offices of general 
Table 1 Distribution of different types of odontogenic cysts $(n=1227)$

\begin{tabular}{lccc}
\hline \multicolumn{1}{c}{ Type of cyst } & \multicolumn{3}{c}{ Frequency } \\
& Number & \% in group & \% in all cysts \\
\hline Developmental & 564 & 100 & 47.4 \\
Dentigerous cyst & 303 & 53.7 & 24.7 \\
Odontogenic keratocyst & 239 & 42.3 & 19.4 \\
Lateral periodontal cyst & 7 & 1.2 & 0.6 \\
Eruption cyst & 7 & 1.2 & 0.6 \\
Gingival cyst & 4 & 0.7 & 0.3 \\
Glandular odontogenic cyst & 4 & 0.7 & 0.3 \\
& & & \\
Inflammatory & 587 & 100 & 46.6 \\
Radicular cyst & 465 & 79.2 & 37.8 \\
Residual cyst & 99 & 16.8 & 8 \\
Paradental cyst & 23 & 3.9 & 1.8 \\
Unclassified & 76 & 100 & 6.0 \\
\hline
\end{tabular}

dentists or specialists (mostly surgeons and radiologists), dental schools and Oral Pathology services, where patients' records and slides are kept for many years. In spite of presenting some biases, files of Oral Pathology diagnostic services are accepted as one of the most valid accessible sources of information on odontogenic lesions and almost all studies on this subject are based on them (6-9).

Little is known about the relative frequency and demographic features of OCs. While some studies have investigated the subject, paucity of information is still obvious $(3,4,6,10-12)$. Such information can not only be regarded as an epidemiologic baseline for further research, but can also be utilized for diagnostic and educational purposes $(7,8,11)$.

Considering the absolute scarcity of information on demographics of OCs in Iran, the aim of this study was to determine the relative frequency of OCs and demographic profile of patients of a major academic Oral and Maxillofacial Pathology center during a 20-year period and to compare the findings with previously published studies.

\section{Materials and Methods}

This retrospective study was approved by the research committee of our university. The data were collected from the archive of the Oral and Maxillofacial Pathology department of the Faculty of Dentistry affiliated to the Tehran University of Medical Sciences. Patient records from 1987 to 2007 (20 years) were reviewed and cases diagnosed as OC were selected. The hematoxylin and eosin-stained histopathologic slides were re-evaluated by both authors to confirm the diagnosis according to WHO classification in 1992 (13). If the final diagnosis could

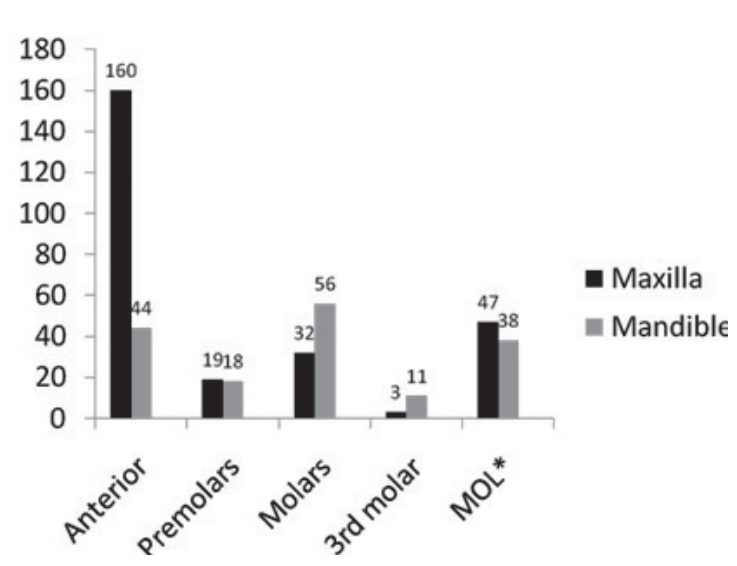

Fig. 1 Site distribution of radicular cysts. *MOL: More than one location involved not be made through histopathologic evaluation alone, clinical and radiographic findings were considered. When the original slides were not representative or were inadequate, new additional slides were prepared from paraffin blocks. In case of controversies, another oral pathologist was consulted in order to ensure that the final diagnosis was correct.

Recurrent lesions, cases in which the diagnosis of odontogenic cysts could not be inferred after these measures, and cases in which the diagnosis changed to an entity other than $\mathrm{OC}$ were excluded from the study. Cases in which a diagnosis of odontogenic cyst could be made based on histopathologic evaluation, but further classification was impossible due to lack of enough clinical and radiographic information, were included in the study. These cases were accepted as unclassified cysts. In approved cases, patients' demographic information (age and sex) and location of the lesion were recorded.

The site of involvement in each jaw was subclassified into four main areas as follows: anterior, premolars, 1st and 2nd molars and 3rd molar/ramus area (in mandibular lesions). For cases involving more than one area shown above, another subcategory defined as "more than one location" (MOL) was considered. Data were analyzed by SPSS software and reported using descriptive statistics.

\section{Results}

From a total of 8529 files reviewed, 1227 cases (14.4\%) met the criteria of OC after histopathologic re-evaluation. 700 cases $(57.2 \%)$ were found in males and 523 cases $(42.8 \%)$ in females. In the remaining cases, the gender of the patients was unknown. The overall male to female ratio was 1.33 . We found 605 cysts in the maxilla (49.6\%) and 615 in the mandible (50.4\%). In 7 cases, the affected 


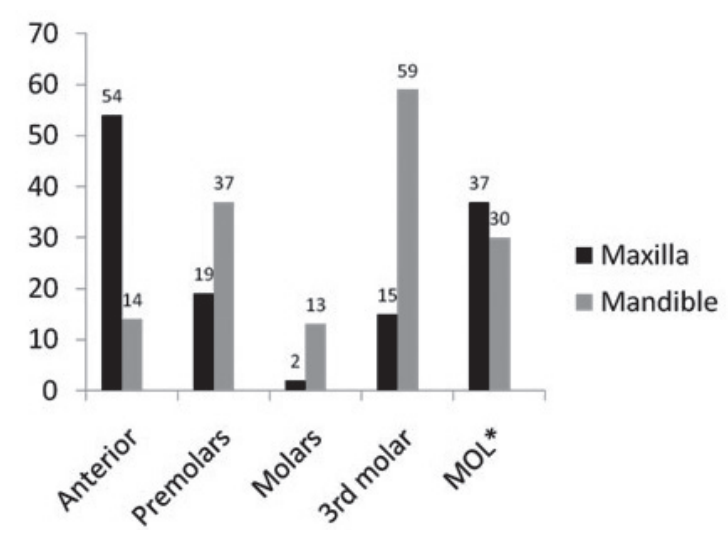

Fig. 2 Site distribution of dentigerous cysts. *MOL: More than one location involved

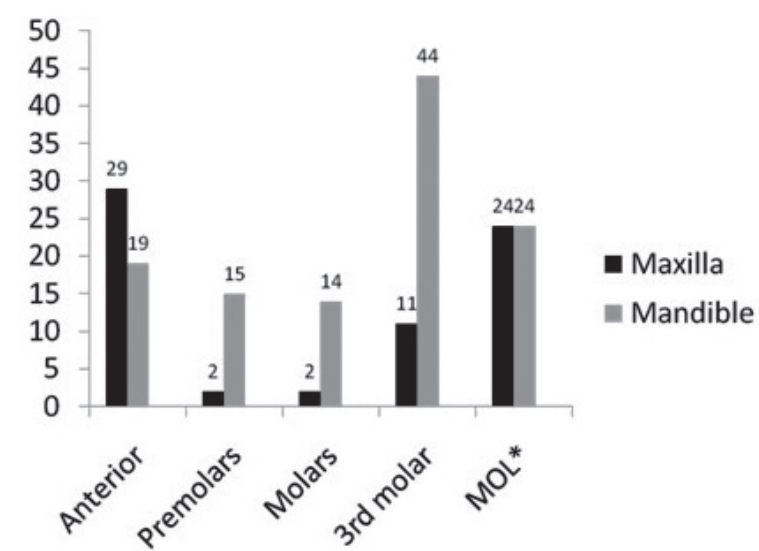

Fig. 3 Site distribution of odontogenic keratocysts. *MOL: More than one location involved

Table 2 Distribution of odontogenic cysts according to sex and age $(n=1227)$

\begin{tabular}{|c|c|c|c|c|c|c|c|c|c|}
\hline \multirow{2}{*}{ Type of Cyst } & \multicolumn{2}{|c|}{ Frequency } & \multicolumn{2}{|c|}{ Male } & \multicolumn{2}{|c|}{ Female } & \multirow[t]{2}{*}{$\mathrm{M} / \mathrm{F}$ ratio } & \multirow{2}{*}{$\begin{array}{l}\text { Age range } \\
\text { (years) }\end{array}$} & \multirow{2}{*}{$\begin{array}{c}\text { Mean } \pm \text { SD } \\
\text { (years) }\end{array}$} \\
\hline & $n$ & $\%$ & $n$ & $\%$ & $n$ & $\%$ & & & \\
\hline Radicular* & 465 & 37.8 & 264 & 56.8 & 201 & 43.2 & 1.31 & 6-84 & $28.7 \pm 12.9$ \\
\hline Dentigerous* & 303 & 24.7 & 173 & 57.3 & 129 & 42.7 & 1.34 & $3-70$ & $21.5 \pm 14.5$ \\
\hline Keratocyst* & 239 & 19.4 & 141 & 59.5 & 96 & 40.5 & 1.46 & $9-78$ & $34.1 \pm 16.4$ \\
\hline Residual* & 99 & 8.0 & 54 & 55.1 & 44 & 44.9 & 1.22 & $11-80$ & $40 \pm 18.0$ \\
\hline Paradental* & 23 & 1.8 & 10 & 43.5 & 13 & 56.5 & 0.77 & $10-48$ & $26.6 \pm 8.2$ \\
\hline Lateral periodontal & 7 & 0.6 & 3 & 42.9 & 4 & 57.1 & 0.75 & $14-54$ & $34.5 \pm 15.0$ \\
\hline Eruption & 7 & 0.6 & 5 & 71.4 & 2 & 28.6 & 2.5 & $7-15$ & $9.8 \pm 2.9$ \\
\hline Glandular* & 4 & 0.3 & 2 & 50 & 2 & 50 & 1 & $28-50$ & $39.0 \pm 15.5$ \\
\hline Gingival & 4 & 0.3 & 1 & 25 & 3 & 75 & 0.33 & $10-57$ & $38.2 \pm 22.5$ \\
\hline Unclassified & 76 & 6.1 & 47 & 61.9 & 29 & 38.1 & 1.62 & $3-67$ & $26.4 \pm 14.1$ \\
\hline Total & 1227 & 100 & 700 & 57.2 & 523 & 42.8 & 1.33 & $3-84$ & $28.6 \pm 15.7$ \\
\hline
\end{tabular}

*In some cases, the age and/or gender was unknown.

jaw was not determined. More than one third of lesions were in the anterior region of the jaws. The patients' age varied from 3 to 84 years with a mean of 28 years. The mean age of male and female patients was 29.1 and 28.0 years, respectively. Sixty-three percent of patients were under 30 years old. The relative frequency of different types of OCs is shown in Table 1.

The most frequent type detected was radicular cyst with a male to female ratio of 1.3. The mean age of patients was 28.8 years (Table 2 ). The lesion was predominantly seen in patients between 10 to 39 years of age (78.2\%). In 428 cases with known site of involvement, maxillary anterior teeth were the most affected. Mandibular molars were the second most affected teeth (Fig. 1).

The second most common type of OC diagnosed was dentigerous cyst, which accounted for 303 cases (24.7\%) with a male to female ratio of 1.3:1. The mean age of patients was 21.5 years and the majority of patients $(80.5 \%)$ were under 30 years of age. In 280 cases, the exact site of presentation was noted. Mandibular $3^{\text {rd }}$ molars/ramus and maxillary anterior teeth were the two most involved regions (Fig. 2).

OKC was the third most common type, which accounted for 239 cases (19.4\%). One hundred forty-one lesions (59\%) were detected in males and $96(40.2 \%)$ in females. In the 2 remaining cases, the gender could not be determined. The mean age of patients was higher in males than in females ( 35 and 32 years, respectively). More than half of the patients $(52.7 \%)$ were in the second and third decades of life. Nearly one third $(31.5 \%)$ of lesions were found in the mandibular third molar/ramus area. Maxillary anterior teeth were the second most common site (Fig. 3).

In 99 cases (7.9\%), a residual cyst was reported. The lesions were almost equally diagnosed in both sexes. About $85 \%$ were distributed evenly between the second and sixth decades of life. The anterior of the jaw was the most frequently involved region.

Twenty-three cases $(1.8 \%)$ of paradental cyst were found and were more frequently diagnosed in females 
Table 3 Different jaw locations of odontogenic cysts

\begin{tabular}{|c|c|c|c|c|c|c|c|c|}
\hline \multirow{2}{*}{$\begin{array}{l}\text { Site } \\
\text { cyst }\end{array}$} & \multicolumn{2}{|c|}{ anterior } & \multicolumn{2}{|c|}{ premolars } & \multicolumn{2}{|c|}{ 1st/2nd molars } & \multicolumn{2}{|c|}{ 3rd molar/ramus } \\
\hline & $n$ & $\%$ & $n$ & $\%$ & $n$ & $\%$ & $n$ & $\%$ \\
\hline Radicular & 205 & 51.3 & 37 & 27.4 & 88 & 61.1 & 14 & 7.7 \\
\hline Dentigerous & 68 & 17.0 & 56 & 41.5 & 15 & 10.4 & 74 & 40.9 \\
\hline Keratocyst & 48 & 12.0 & 17 & 12.6 & 16 & 11.1 & 57 & 31.5 \\
\hline Residual & 37 & 9.3 & 10 & 7.4 & 13 & 9.0 & 2 & 1.1 \\
\hline Unclassified & 25 & 6.3 & 5 & 3.7 & 6 & 4.2 & 12 & 6.6 \\
\hline Others & 17 & 4.1 & 10 & 7.4 & 6 & 4.2 & 22 & 12.2 \\
\hline Total & 400 & 100 & 135 & 100 & 144 & 100 & 181 & 100 \\
\hline
\end{tabular}

and mostly in the second and third decades of life (77\%). Mandibular third molar/ramus area was the most prevalent site of presentation.

Lateral periodontal cyst, eruption cyst, glandular odontogenic cyst and gingival cyst of adults were also diagnosed. These OCs were predominantly located in the mandible. A total of 76 cases $(6 \%)$ remained unclassified. Out of these, 67 cases showed microscopic evidence of an inflammatory process (Tables 1 and 2).

Jaw involvement in the most prevalent cyst types is shown in Table 3. The anterior and the first two molar areas were predominantly affected by radicular cyst. Lesions in premolars and third molar/ramus regions were mostly diagnosed as dentigerous cyst or OKC.

\section{Discussion}

Epidemiologic studies are primarily based on population-based surveys. However, in rare diseases, registries and archives of main academic and/or medical centers provide precise data and are the only accepted source of data collection upon which many important studies are based. Some investigators have used the terms "prevalence" or "incidence" to describe the results obtained from these records $(4,6,12)$. We prefer to use "relative frequency" because the aforementioned terms should only be used in population-based surveys, and otherwise could be misleading (14).

The classification of OCs has been controversial for many years. In the most recent WHO classification, $\mathrm{OKC}$ is no longer considered as a cystic entity and is now entered in the neoplastic category by the name of "keratocystic odontogenic tumor" (15). However, Shear and Speight (1) as well as Neville et al. (2) still regard the lesion as an odontogenic cyst of developmental origin. The authors share the views of Shear and Speight and Neville et al. Also, in order to compare our findings with previous articles, OKC is included in our investigation.

Among a total of 8529 biopsy specimens received over the 20-year period between 1987 and 2007, 1227
OCs were diagnosed, which constitutes $14.4 \%$ of all specimens. This is identical to some studies carried out in Brazil (3), Singapore (16) and India (17), which reported frequencies of $14.7 \%, 14.9 \%$ and $14.3 \%$, respectively, and is similar to those from England (12.8\%) (7) and Mexico (11.5\%) (8). This was more than what was reported in Chile (10\%) (9), Italy (10.4\%) (12) and another study from Mexico (7.8\%) (10), and is relatively less compared to those from the US (19.4\%) (18) and Canada (17.2\%) (6). From the current literature, it could be concluded that OCs make up $0.8 \%$ to $45.9 \%$ of all biopsied lesions $(3,7)$. These variations may be primarily due to differences in attitude and practice toward performing biopsies and sending specimens for histopathologic evaluation. Possible biases, different sources and methods of sampling and other epidemiologic factors should also be considered, which in turn highlight the necessity of such research worldwide.

The overall male to female ratio in the present study was $1.33: 1$, which is identical to what was found in Mexico (10) and is similar to the results of Jones et al. in England (1.27:1) (7). From the literature, it could be concluded that OCs are more prevalent in men than in women.

In our study, the most frequently diagnosed lesions were radicular cyst, dentigerous cyst and OKC. This is in complete accordance with other studies (6-12). However, the order is different in pediatric patients $(19,20)$.

Radicular cysts, the most common of OCs, develop as a result of pulpal necrosis following pulpitis and thus have an inflammatory origin. In this study, they accounted for $37.9 \%$ of the lesions. Most similar studies reported a relative frequency between 47 to $58 \%(7,9,11,18,19)$. In few studies, it was more than $60 \%(3,6)$. Our finding is in close agreement with studies from Mexico $(8,10)$ and Jordan (4), which reported a frequency of nearly $40 \%$. Many clinicians believe that periapical pathoses can be readily diagnosed through clinical and radiographic examinations. This, together with the relatively higher 
prevalence of these lesions compared to other similar entities, may be the reason for the reluctance to perform biopsy or to send periapical tissues associated with the extracted carious teeth for histopathologic evaluation. Although not professionally acceptable, this can account for the comparably lower figure.

We found radicular cysts 1.3 times more often in men than in women, which corresponds to other studies (8). Nearly $60 \%$ of radicular cysts were diagnosed in patients between 10 to 30 years old. This together with the mean age of patients indicate a much earlier age of occurrence in this study compared to other studies $(3,7,9,11,12)$. Simple caries preventive measures along with easier and more accessible oral health care services may help prevent or postpone these inflammatory lesions. As in previously published studies, they were predominantly observed in the anterior maxillary region $(3,4,7,9,10,12)$. Aesthetic reasons may account for people's eagerness to conserve carious teeth in this region for a longer time, upon which chronic inflammation incites cystic degeneration in periapical tissues (9).

Dentigerous cyst, accounting for $24.7 \%$ of cases, was the second most frequent lesion in this study. The entity is characteristic for its radiographic view; a well-defined lucency surrounding the crown of an unerupted tooth. The relative frequency is reported to be between 15 to $25 \%$ in the literature $(3,4,6,7,9,11,19)$. The male to female ratio found here was identical to that in the study of Mosqueda et al. (9) and in agreement with other investigations for male predominance $(4,9-12)$. The peak incidence was in the first two decades of life, which parallels findings of Ochsenius et al. (9) and Ledesma-Montes et al. (10), but seems to be lower compared to studies reporting the peak in the second and third decades $(3,4)$ and the fifth and sixth decades (7). In accordance with the literature, mandibular third molars and maxillary anterior teeth were the two main sites of presentation. However, other regions were also reported, which may account for the lower age at the time of diagnosis in the present study.

OKCs are known as lesions imitating a wide range of cystic and neoplastic lesions in radiographic views. However, they exhibit several histopathologic hallmarks that can lead to positive diagnoses. Nineteen percent of cases in our study satisfied the criteria for OKC. The figure is identical to studies from Mexico (10) and France (11), but is greater compared to the majority of other similar studies which reported a relative frequency from $1.3 \%$ to $11.6 \%(3,4,6,7,12)$. The predilection of the lesion to occur more in male patients, in the posterior mandibular region and in the second and third decades of life in the present study is in agreement with the literature
$(3,4,7-12,21,22)$. Like other investigations $(9,23)$, the maxillary anterior region was the second most prevalent site of presentation for OKC in our study. In this region, as Ali and Baughman have documented, there is a high risk that $\mathrm{OKC}$ be misdiagnosed for inflammatory lesions like radicular cyst or granuloma (23). The recurrence rate for $\mathrm{OKC}$ in this area has been reported to be as high as $70 \%$ (22). These findings suggest that the anterior maxilla is a critical region where the need for histopathologic examination of periapical radiolucencies should be stressed.

The recurrence rate of odontogenic keratocyst has been extensively studied. Regarding variations in number of patients and duration of follow-up examinations, recurrence rates from $18 \%$ to $58.3 \%$ have been reported $(21,22)$. In our country, follow-up examinations are not routinely performed and recorded; therefore, no systematic data concerning the recurrence rate could be retrieved from the files. We excluded sporadic cases of recurrence from the final analysis in order to obtain more uniform data.

Multiple cases of OKC have been reported in the literature that could be associated with Gorlin syndrome. The main focus of this study was odontogenic cysts as separate entities not associated with other diseases. Also, it should be noted that patient records in our institution are not computerized and as mentioned above, follow-up examinations are not routinely performed. The possibility that patients may visit different centers for recurrent or multiple lesions should be considered. Therefore, we could not draw any conclusion regarding the prevalence of this syndrome among our cases.

Residual cyst is analogous to radicular cyst; after the causal tooth is extracted, the remaining cyst is called a residual cyst. In this study, they comprised $8 \%$ of all diagnoses, which is identical to a study from UK (7) and is similar to other studies $(9-11,18)$. Our findings on gender, site and age distribution of the lesion agreed with other studies providing detailed information $(7,9)$.

Paradental cyst, another OC of inflammatory origin, is mainly associated with partially erupted mandibular third molars previously involved in pericoronitis. The relative frequency of the lesion in the literature varied from less than $1 \%(3,6,19)$ to $5.6 \%(7)$. Twenty-three cases were paradental cysts, all were in the mandible and almost all were associated with the third molars. Although paradental cysts are rare entities, those arising from teeth other than mandibular third molars are even scarcer $(7,9,24,25)$. The literature supports our findings that the majority of paradental cysts are diagnosed in the third decade of life $(7,24)$, the time of eruption for 
the mandibular third molars especially if delayed as in partially erupted ones.

The preponderance of the lesion in females found in this study is in accordance with other studies $(3,24)$, but is in contrast with studies that reported a firm male predilection (8). There does not seem to be a positive gender predilection in this entity, and as Ackermann et al stated: "there is no known difference between males and females in the incidence of impaction or pericoronitis" (26).

Based on our findings, the distribution pattern of OCs in Iran is relatively similar to studies from other parts of the world. The importance of collecting adequate clinical information regarding final diagnosis of these lesions and performing routine follow-up examination is further emphasized.

\section{Acknowledgments}

The authors wish to thank Mrs. Nahid Hajesmail for technical assistance and slide preparation.

\section{References}

1. Shear M, Speight P (2007) Cysts of the oral and maxillofacial regions. 4th ed, Blackwell, Munsgaard, 1-4.

2. Neville B, Dam D, Allen C, Bouquot J (2009) Oral and maxillofacial pathology. 3rd ed, WB Saunders, 678-700.

3. Grossmann SM, Machado VC, Xavier GM, Moura MD, Gomez RS, Aguiar MC, Mesquita RA (2007) Demographic profile of odontogenic and selected nonodontogenic cysts in a Brazilian population. Oral Surg Oral Med Oral Pathol Oral Radiol Endod 104, e35-41.

4. Bataineh AB, Rawashdeh MA, Al Qudah MA (2004) The prevalence of inflammatory and developmental odontogenic cysts in a Jordanian population: a clinicopathologic study. Quintessence Int 35, 815-819.

5. Muglali M, Sumer AP (2008) Squamous cell carcinoma arising in a residual cyst: a case report. J Contemp Dent Pract 9, 115-121.

6. Daley TD, Wysocki GP, Pringle GA (1994) Relative incidence of odontogenic tumors and oral and jaw cysts in a Canadian population. Oral Surg Oral Med Oral Pathol 77, 276-280.

7. Jones AV, Craig GT, Franklin CD (2006) Range and demographics of odontogenic cysts diagnosed in a UK population over a 30 -year period. J Oral Pathol Med 35, 500-507.

8. Mosqueda-Taylor A, Irigoyen-Camacho ME,
Diaz-Franco MA, Torres-Tejero MA (2002) Odontogenic cysts. Analysis of 856 cases. Med Oral 7, 89-96.

9. Ochsenius G, Escobar E, Godoy L, Penafiel C (2007) Odontogenic cysts: analysis of 2,944 cases in Chile. Med Oral Patol Oral Cir Bucal 12, E85-91.

10. Ledesma-Montes C, Hernandez-Guerrero JC, Garces-Ortiz M (2000) Clinico-pathologic study of odontogenic cysts in a Mexican sample population. Arch Med Res 31, 373-376.

11. Meningaud JP, Oprean N, Pitak-Arnnop P, Bertrand JC (2006) Odontogenic cysts: a clinical study of 695 cases. J Oral Sci 48, 59-62.

12. Tortorici S, Amodio E, Massenti MF, Buzzanca ML, Burruano F, Vitale F (2008) Prevalence and distribution of odontogenic cysts in Sicily: 19862005. J Oral Sci 50, 15-18.

13. Kramer IR, Pindborg JJ, Shear M (1992) Histological typing of odontogenic tumors. 2nd ed, World Health Organization, Geneva.

14. Speechley M, Stephens R (1995) What is "relative incidence" ? Oral Surg Oral Med Oral Pathol Oral Radiol Endod 79, 2-3.

15. Philipsen HP (2005) Keratocystic odontogenic tumor. In: World Health Organization classification of tumours. Pathology and genetics of head and neck tumours, Barnes L, Eveson JW, Reichart P, Sidransky D eds, IARC Press, Lyon, 306

16. Tay AB (1999) A 5-year survey of oral biopsies in an oral surgical unit in Singapore: 1993-1997. Ann Acad Med Singapore 28, 665-671.

17. Das SN, Brave VR, Shetty RP (1994) A survey of 4478 biopsy specimens of oral lesions. J Pierre Fauchard Acad 8, 143-147.

18. Bhaskar SN (1968) Oral pathology in the dental office: survey of 20,575 biopsy specimens. J Am Dent Assoc 76, 761-766.

19. Kreidler JF, Raubenheimer EJ, van Heerden WF (1993) A retrospective analysis of 367 cystic lesions of the jaw - the Ulm experience. J Craniomaxillofac Surg 21, 339-341.

20. Shah SK, Le MC, Carpenter WM (2009) Retrospective review of pediatric oral lesions from a dental school biopsy service. Pediatr Dent 31, 14-19.

21. Chirapathomsakul D, Sastravaha P, Jansisyanont P (2006) A review of odontogenic keratocysts and the behavior of recurrences. Oral Surg Oral Med Oral Pathol Oral Radiol Endod 101, 5-9.

22. Myoung H, Hong SP, Hong SD, Lee JI, Lim CY, 
Choung PH, Lee JH, Choi JY, Seo BM, Kim MJ (2001) Odontogenic keratocyst: review of 256 cases for recurrence and clinicopathologic parameters. Oral Surg Oral Med Oral Pathol Oral Radiol Endod 91, 328-333.

23. Ali M, Baughman RA (2003) Maxillary odontogenic keratocyst: a common and serious clinical misdiagnosis. J Am Dent Assoc 134, 877-883.

24. de Sousa SO, Correa L, Deboni MC, de Araujo VC (2001) Clinicopathologic features of 54 cases of paradental cyst. Quintessence Int 32, 737-741. 25. Philipsen HP, Reichart PA, Ogawa I, Suei Y, Takata $T$ (2004) The inflammatory paradental cyst: a critical review of 342 cases from a literature survey, including 17 new cases from the author's files. J Oral Pathol Med 33, 147-155.

26. Ackermann G, Cohen MA, Altini M (1987) The paradental cyst: a clinicopathologic study of 50 cases. Oral Surg Oral Med Oral Pathol 64, 308-312. 